\title{
Improving Source Selection in Large Scale Mediation Systems through Combinatorial Optimization Techniques
}

\author{
Alexandra Pomares ${ }^{1}$, Claudia Roncancio ${ }^{2}$, Van-Dat $\mathrm{Cung}^{2}$, and \\ María-del-Pilar Villamil ${ }^{3}$ \\ 1 Pontificia Universidad Javeriana, Bogotá, Colombia \\ ${ }^{2}$ Grenoble INP, Grenoble, France \\ 3 Universidad de los Andes, Bogotá, Colombia
}

\begin{abstract}
This paper concerns querying in large scale virtual organizations. Such organizations are characterized by a challenging data context involving a large number of distributed data sources with strong heterogeneity and uncontrolled data overlapping. In that context, data source selection during query evaluation is particularly important and complex. To cope with this task, we propose OptiSource, an original strategy for source selection using combinatorial optimization techniques combined to organizational knowledge of the virtual organization. Experiment numerical results show that OptiSource is a robust strategy that improves the precision and the recall of the source selection process. This paper presents the data and knowledge models, the definition of OptiSource, the related mathematical model, the prototype and an extensive experimental study.
\end{abstract}

Key words: Large Scale Data Mediation, Source Selection, Combinatorial Optimization

\section{Introduction}

Source Selection (also known as server or database selection) is the process of identifying the set of data sources that must participate in the evaluation of a query. Source selection is particularly critical in large scale systems where the number of possible query plans grows rapidly as the complexity of the query and the set of data sources increases. Research in this area has been promoted mainly by the requirements of the world wide web, distributed file sharing systems and distributed databases.

This paper explores the problem of source selection in large scale data contexts that are typical of the new type of organizational model called Virtual Organization (VO). They combine characteristics of web data contexts, data heterogeneity and high distribution, and characteristics of enterprise data contexts, like availability commitment and structured data sources. 


\subsection{Querying in Large Scale Virtual Organizations}

A Virtual Organization (VO) is a set of autonomous collaborating organizations, called VO units, working towards a common goal. It enables disparate groups to share competencies and resources such as data and computing resources [1]. VOs work in a domain (e.g. health) and their participants share information about objects of interest in the domain (e.g. patients, clients). Instances of those types of objects have to be composed from fragments distributed in the data sources of the VO participants. Data may be partially replicated on several sources. Considering a type of object, an important type of query is the selection of the instances satisfying a given condition (a selection predicate). For example, considering the type Patients, a query seeking those such as gender $=$ female and diagnosis $=$ cancer may potentially involve querying all data sources having a fragment of a Patient. However, if a complete answer is not required (e.g. the user is doing a research that does not need all the patients, but a good sample), the query optimizer may use heuristics to prune some data sources in order to speed up the response time of the query.

\subsection{Challenges in Source Selection in VO Data Contexts}

Source selection and data integration are particularly challenging in VO data contexts. As VOs have evolved to national and world-wide magnitudes [2,3], it is unlikely that VO participants provide precise metadata describing the content of their data sources and if they do maintain such metadata, it would be costly. Furthermore, due to the heterogeneity of data sources, data may be provided with different levels of details making source selection harder. For example, to look for patients having a cancer, the system has to be able to recognize that sources having data of patients with breast cancer or invasive lobular carcinoma are relevant. A deep analysis between metadata and queries is therefore required to come out with an effective query plan.

Another challenge is introduced by the uncontrolled data overlapping between data sources. An instance of a subject may exist in several data sources which may or may not have overlapping sets of properties. Information may be replicated without having necessarily "well formed copies". This prevents the use of strategies that consider data sources are complete or independent [4].

The aforementioned characteristics of the data context may be a consequence of business processes in the VO. Heterogeneity, source overlapping, large distribution and a high number of data sources make query processing a complex task, specially in the planning phase. Several existing proposals on source selection take into account distribution, heterogeneity and integration of structured data sources $[5,6,7,8]$. Nevertheless, as shown in the following, they are not well suited for VOs, some of them can be very inefficient or simply cannot be used in VO data contexts. The main reasons are that such proposals do not address uncontrolled replication and non-disjoint fragments, or because their requirements of data source metadata are not fulfilled in VOs. 


\subsection{Contribution and Paper Organization}

The work presented in this paper is a contribution to improve source selection in large scale virtual organizations having a complex data context. It presents OptiSource, a source selection strategy combining two original aspects:

- The first one is the introduction of roles for data sources based on knowledge associated to the VO. A seamless integration of such roles with the taxonomy of concepts used in the VO permits a good estimation of the relevance of using a data source to evaluate a query.

- The second one is the use of a combinatorial optimization model to control the number of sources to contact. The objective function maximizes the benefit of using a data source and minimizes the number of sources to contact while satisfying processing resource constraints. The efficiency of source selection is improved so as its recall and precision criteria.

To the best of our knowledge there are not yet other proposals using combinatorial optimization techniques in conjunction with ontologies during query evaluation. Our research shows that this approach is promising.

This paper presents the full proposal of OptiSource, its definition, a prototype and a performance study. It is an extension of the work presented in [9]. It includes a deep analysis of related works, it provides a detailed presentation of each component of OptiSource, and it analyzes the complexity of the mathematical model used to optimize the assignment of data sources to query conditions. In addition, it presents in detail the experimental environment and reports additional tests and analyses about the impact of prediction accuracy during the process of source selection.

In the following, Section 2 presents the terminology and notation used in the paper. Section 3 gives an analysis of related works in source selection and the utility of existing proposals in large scale VOs. Section 4 presents OptiSource, the source selection strategy we propose. Section 5 presents the combinatorial optimization model used in OptiSource. Section 6 presents the OptiSource prototype. A large variety of tests have been executed to evaluate our proposal. The results are discussed in Sections 6 and 7. Finally, Section 8 presents our conclusions and research perspectives.

\section{Preliminaries}

The source selection strategy proposed in this work is part of a mediation system for large scale VOs. At the mediation level data are represented as Virtual Data Objects (VDO) which link together several concepts.

Definition 1 Virtual Data Object (VDO). A VDO is a logical set of related concepts relevant to a group of users. Figure 1 illustrates a VDO joining four concepts. Each concept has data type properties whose values are data literals. Concepts are related through object type properties whose values are instances. VDOs are virtual because their instances are partitioned and distributed on several data sources. 


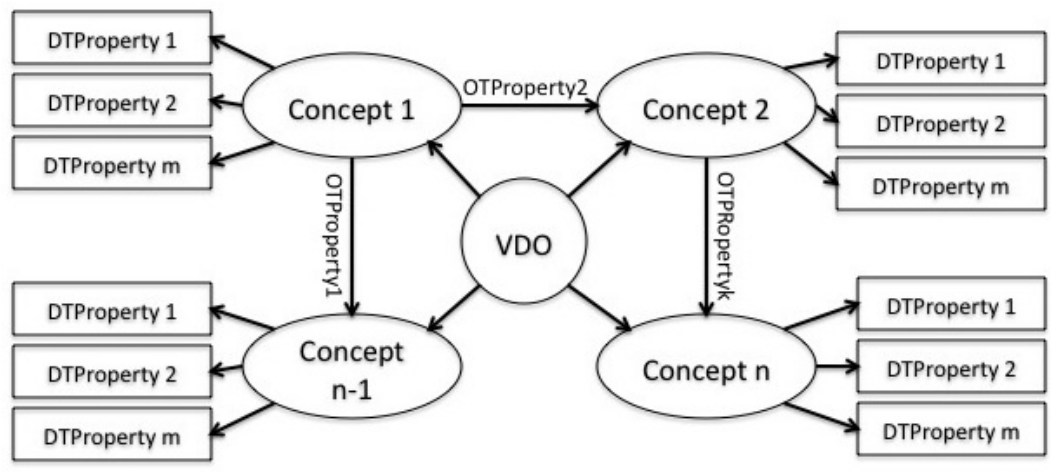

Fig. 1. Virtual Data Object.

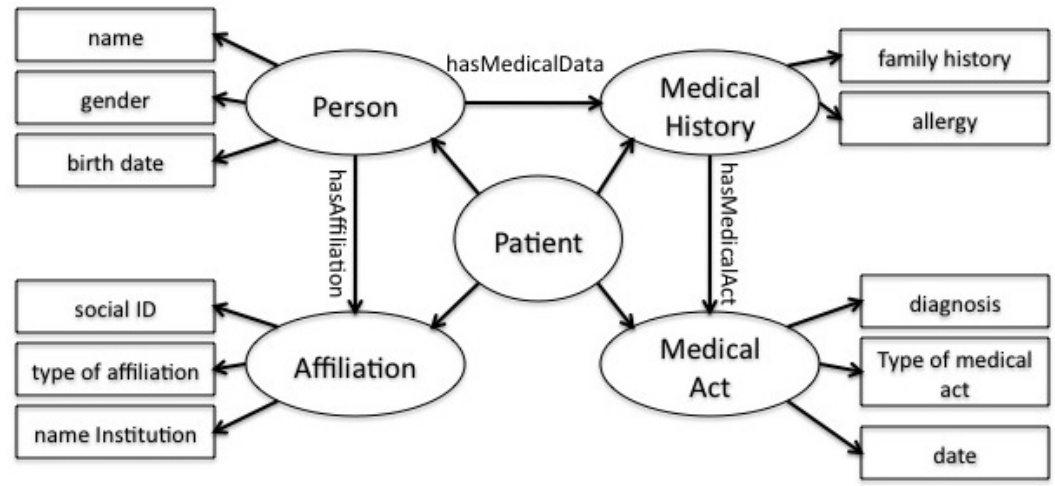

Fig. 2. VDO Patient.

Figure 2 shows a VDO Patient. Four concepts (Person, Medical Act, Medical History, Affiliation) are related to compose the VDO Patient.

We consider here queries with selection conditions on the properties of the concepts composing the VDO. For example, the query hereafter uses the VDO Patient and selects the id of patients with a "Buphtalmos" diagnosis.

Query $1 Q\left(V D O_{\text {Patient }}, \operatorname{properties}(i d)\right.$, conditions $($ diagnosis $=$ Buphtalmos $\left.)\right)$

In the following we will work with queries including several conditions as follows:

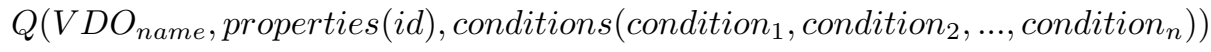

Data abstraction levels

We consider an enriched architecture of mediation with three levels of data abstraction (see Figure 3 for an example). The external level presents the set 


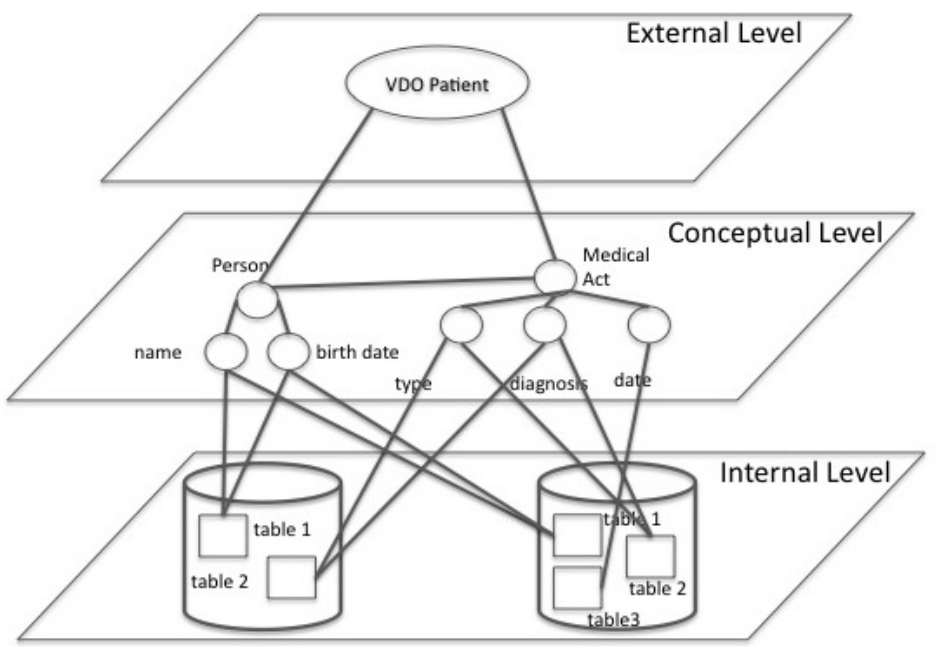

Fig. 3. Data Abstraction Levels.

of VDOs supported by the system. Queries will refer to them. The conceptual level contains the reference ontology that defines the domain of the VO. The concepts defined in this ontology are used to create VDOs. Finally, the internal level contains all the data sources available in the VO. Such data is used to build the instances of the VDO concepts.

The rest of the paper will be focused on the strategy used to identify the data sources from the internal level that must be used to evaluate the query defined at the external level.

\section{Data Source Selection: Related Works}

Even though multi-sources querying has been intensively studied for more than 15 years, none of the available strategies for query planning can be directly applied for large scale VO data contexts.

This section presents an analysis of the main source selection strategies available in the literature in order to identify interesting ideas that could be applied on large scale VO contexts. We analyze several proposals related to source selection including structured, semi-structured and non structured sources as seen in Figure 4. However, in this paper we consider the most representative proposals such as capability, multi-utility and quality oriented systems.

\subsection{Capability Oriented Systems}

In the first generation of integration systems for structured sources like Information Manifold [10], TSIMMIS [11], DISCO [12] and Minicon [8], source selection 


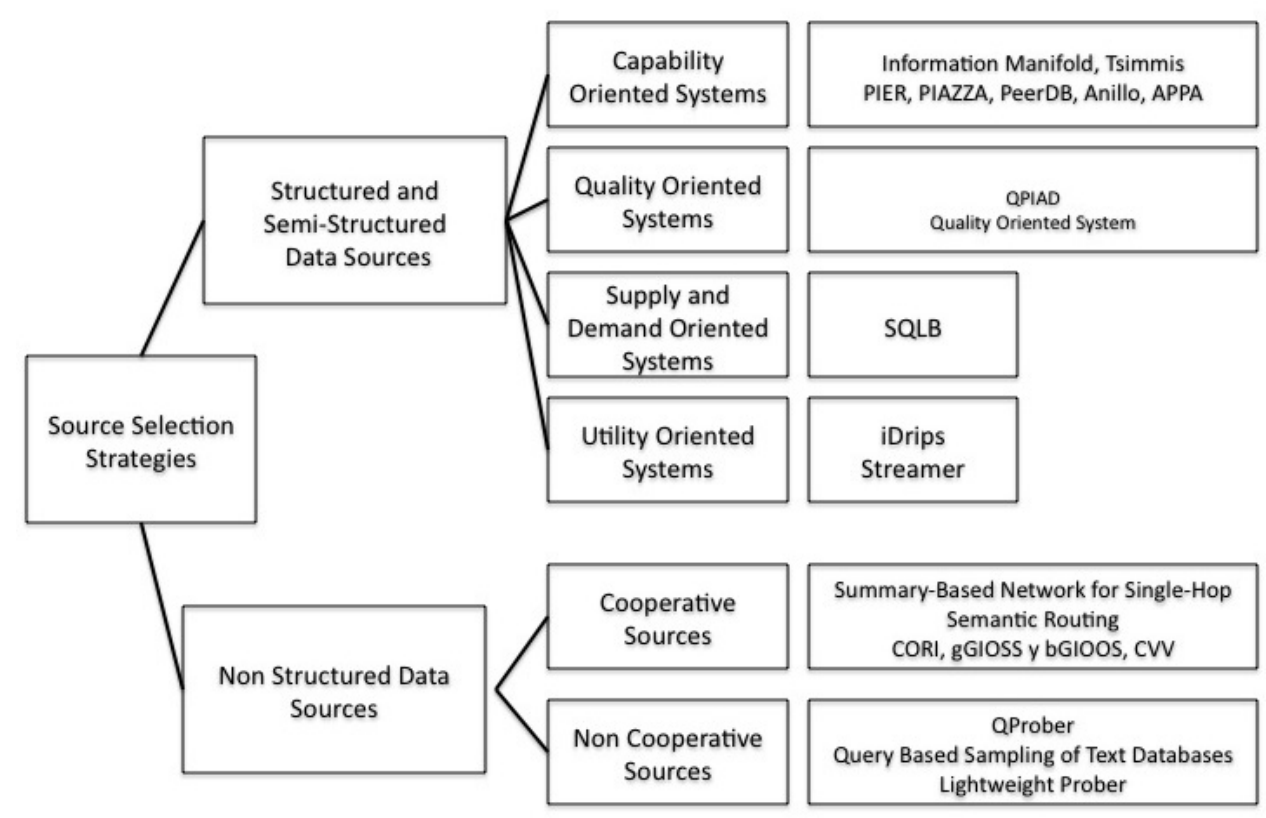

Fig. 4. Source Selection Strategies.

is performed according to the capabilities of sources. These capabilities are associated to the capacity to evaluate types of queries, for example the number of conditions and the attributes that can be restricted in a query. In this way, they require knowledge about the intentional level and the computing capacity [13] (e.g. which attributes can be used as filters) of each data source.

Although such proposals are appropriate in several contexts, they are not able to reduce significantly the set of data sources that must participate to evaluate a query in a VO because the processing capabilities of sources are typically alike and, as a consequence, are not useful to differentiate data sources.

\subsection{Multi-utility Oriented Systems}

Another group of proposals like iDrips and Streamer [5] conceive the source selection as the identification of most useful sources for executing a query. These sources are selected comparing utility functions of different plans for executing the query.

iDrips and Streamer assume source similarity property. Source Similarity means that similar sources can be replaced one by another without affecting the utility of a plan. Whereas Plan Independence means that two plans are independent if the utility of a plan does not depend on whether the other has been executed. 
iDrips algorithm acts in two phases: the first phase groups similar data sources and creates abstract plans that are capable of solving all the predicates of the query. Each abstract plan has an utility and represents a set of concrete plans.

The second phase obtains query plans in decreasing order of utility. It is decomposed into iterations. Each iteration selects the most dominant abstract plan, removes it from the plans space, and rebuilds the dominance relationships that are necessary. A dominant abstract plan contains at least one concrete plan that maximizes the utility in comparison to all concrete plans in other abstract plans.

The Streamer algorithm is a modification of iDrips. Streamer assumes that plans are independent avoiding to recalculate the dominance relationships in each iteration.

The effectiveness of these algorithms depends on the utility function. Several utility functions are proposed to represent the relationships between data sources. However, with the coverage utility function, used in the paper [5], it is not clear how to obtain variables involved in its calculation. Additionally, even though the coverage of query plans could be obtained, it does not consider the extensional overlapping between sources incurring on inefficiencies during the ordering. This lead to restrict the use of the algorithm on VO contexts where utility functions must be able to express relationships between sources like overlapping and replication.

\subsection{Quality Oriented Systems}

More recent works like the strategy proposed in Bleiholder et al. [14] assume the source selection as the identification of the better paths between overlapped data sources to resolve a query. These paths maximize the benefit in terms of the number of objects obtained in the answer, while respecting a cost constraint or budget limitations.

They model the problem of selecting the best set of paths as a dual problem of budgeted maximum coverage (BMC) and maximal set cover with a threshold (MSCT). These problems were solved through three types of algorithms: integer programming/linear programming algorithm, greedy algorithms and a random search algorithm.

According to BMC problem, greedy algorithms are useful only with higher budgets; whereas linear programming algorithms and the random algorithm have good behavior with different levels of budgets. The MSCT problem is solved only with greedy algorithms, which do not always find the optimal solution. This proposal is very efficient in data contexts where it is possible to know the instances that are contained in each data source. However, assumptions of this kind of detailed knowledge about data sources is unavailable in most of the distributed data contexts. Consequently, the application of the proposed model in this kind of contexts is not feasible. The strategies to obtain the relationships between objects of data sources and the benefit of each path should be provided in order to enable the use of these algorithms in any context. 
Other proposals like QPIAD [6] and Quality Oriented system [15] use detailed statistics on data source quality to reduce the number of possible query plans and therefore sources. QPIAD is a rewriting strategy that allows to retrieve relevant answers from autonomous data sources, using correlations between attributes used on predicates of a query. These answers are incomplete because they include answers obtained using query rewritings where some conditions will be replaced, in particular using null values. For instance, a query with model=Accord and make $=$ Honda conditions can be rewriting as model $=$ Accord and make $=$ null, generating incomplete answers.

QPIAD principle is to learn attribute correlation (e.g. make and model), value distribution (e.g. Honda and Accord) and query selectivity in order to retrieve incomplete answers that have a high probability of being certain answers.

The QPIAD algorithm acts in two phases: the first one sends the original query to all the available data sources to obtain the set of certain answers. These answers are sent to the final user. The second phase learns approximate correlations between attribute values, using the set of certain answers and a sample of data sources that was previously built. These correlations are used to generate the rewritten queries that are sent to data sources to obtain possible answers. Although these queries may retrieve possible answers they do not have the same relevance. Consequently, the algorithm ranks the queries using their estimated precision and recall, and then QPIAD sends them to the data sources. Only the first $\mathrm{k}$ queries are sent to the data sources. The value of $\mathrm{k}$ is the maximum number of queries supported by each data source.

QPIAD allows to obtain complete answers in data contexts where data sources have quality problems. This strategy includes an innovative algorithm to learn approximate functional dependencies between attribute values that can be useful not only in source selection problems, but also in data mining projects. However, the rewriting phase of QPIAD in large scale distributed data contexts leads to scalability problems. Even though the queries defined by QPIAD improves the quality of the result, it is heavily resource dependent. The algorithm of ranking of QPIAD can include a phase of analysis of data sources in order to predict which data sources are more relevant for the query.

Finally, proposals using a P2P approach (PIER [7], PIAZZA [16], EDUTELLA [17], SomeWhere [18]) manage numerous sources. However, as their rewriting and source selection strategies do not take into account source overlapping, they would lead to high redundancy in the answers when used in large scale VOs.

\section{OptiSource: a Decision System for Source Selection}

The analysis of related works led us to identify the lack of a strategy that assures scalability of the query evaluation w.r.t. the number of available data sources when there are overlapping and uncontrolled replication between data sources. This gap, critical for the mediation in large scale data contexts, is fulfilled with OptiSource, a strategy of source selection created for large scale VOs. This 
section presents OptiSource, its principle and its general process in Section 4.1 and its components in Sections 4.2, 4.3, 4.4, 4.5.

\subsection{Principle and General Process}

In order to enable scalability, OptiSource principle is to identify for each condition the minimal set of sources required to obtain the maximum number of instances that match the condition, while avoiding the contact of unnecessary sources. The minimal set of data sources is identified selecting iteratively the most profitable data sources w.r.t. the query. The profit of data sources w.r.t. a query reflects the estimated benefit of using a data source to evaluate a query and is calculated using the aspects presented in Table 1 . The data sources whose estimation of benefit is higher are considered the most profitable. The first iteration selects the data sources with higher benefit w.r.t. each condition of the query. The subsequent iterations remove the sources selected in previous iterations and identify the next most profitable data sources. The iterations continue until the user is satisfied with the number of obtained instances or until there is no more available data sources.

Table 1. Profitability aspects.

\begin{tabular}{|l|l|}
\hline Aspect & Description \\
\hline Intentional matching & $\begin{array}{l}\text { Identify which sources contain VDO properties re- } \\
\text { quired in the query }\end{array}$ \\
\hline Extensional matching & $\begin{array}{l}\text { Identify which data sources contain instances that } \\
\text { match all or part of the predicate of the query, and } \\
\text { from these which will better contribute in terms of in- } \\
\text { stances that match the predicate }\end{array}$ \\
\hline Data Source compatibility & $\begin{array}{l}\text { Due to the incompleteness of data sources, in order to } \\
\text { avoid efforts of integration of data sources that do not } \\
\text { contain instances in common, it must identify the sets } \\
\text { of data sources whose join operation will not produce } \\
\text { an empty set of instances }\end{array}$ \\
\hline Data Source redundancy & $\begin{array}{l}\text { Identify which data sources can be filtered due to its } \\
\text { redundancy in terms of instances with other sources }\end{array}$ \\
\hline
\end{tabular}

The principle of OptiSource is materialized in an iterative process whose inputs are the $n$ available data sources $D S i$ with their mapping (or view) $M_{i}$ to the VDO (e.g. Person, MedicalAct) and the conditions $p_{j}$ involved in the query predicate:

$$
\text { Input }=\left[\left(D S 1, M_{1}\right),\left(D S 2, M_{2}\right), \ldots,\left(D S n, M_{n}\right)\right],\left[p_{1}, p_{2}, \ldots, p_{m}\right] .
$$

The output of the process is the collection of most profitable joining sets. A joining set is defined as a group of data sources able to evaluate all the conditions of the query predicate and whose join operation between the data sources it comprises will not produce an empty set of instances.

$$
\text { Output }=\left(J S_{1}, J S_{2}, \ldots, J S_{p}\right) .
$$




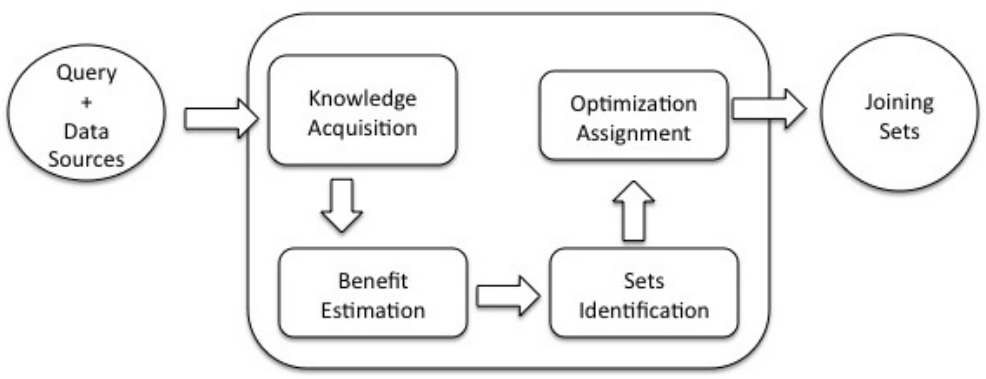

Fig. 5. Activities of OptiSource.

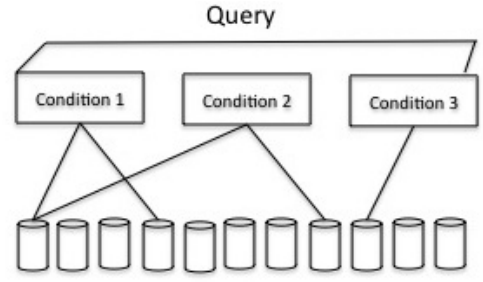

Joining Set 1

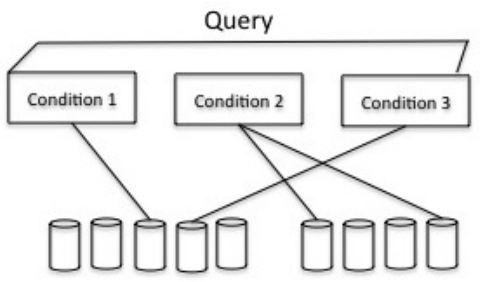

Joining Set 2

Fig. 6. OptiSource Output.

where $J S_{i}=\left(D S_{k}, p_{1}\right), \ldots,\left(D S_{l}, p_{m}\right), 1 \leqslant k, l \leqslant n$. A couple $\left(D S_{k}, p_{j}\right)$ means that the data source $D S_{k}$ will evaluate the condition $p_{j}$. A source can evaluate several conditions $p_{j}$ and a condition $p_{j}$ can be evaluated by several sources.

Figure 5 presents the general activities of the process conducted by OptiSource. First, OptiSource queries a dynamically updated knowledge base that contains the facts describing the characteristics of data sources. With the acquired knowledge it estimates the benefit of using each data source to evaluate the query and identifies the sets of data sources with high tendency to share instances. Next, using the estimated benefit it optimizes the assignment of conditions within each set of data sources. At the end, the output are the joining sets identifying the conditions that each data source will evaluate. Figure 6 presents an example of the output of the process.

\subsection{Components of OptiSource}

The activities of OptiSource are executed by the components presented in Figure 7. The Selection Coordinator is in charge of controlling the execution of the activities to produce the output of the source selection process. The Knowledge Administrator is responsible for controlling the interaction with the VO Knowledge Base, it queries the base and provides the other components with the knowledge facts it obtains. The Benefit Estimator uses the VO Knowledge 


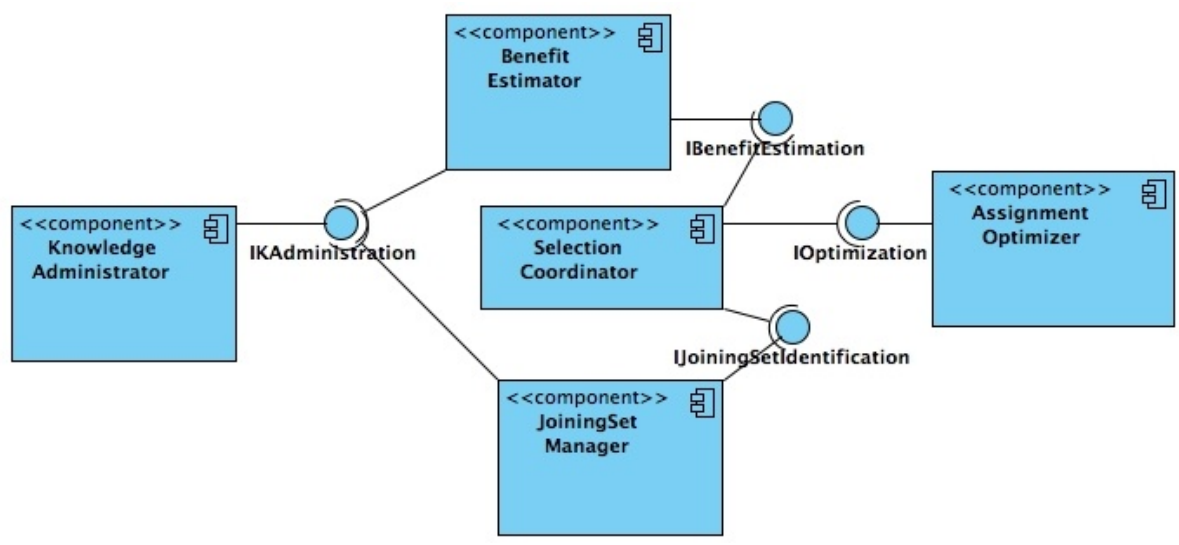

Fig. 7. OptiSource Components.

Base facts to predict the benefit of using a data source to evaluate the conditions of a query predicate The Joining Set Manager determines the sets of data sources that can work together in order to evaluate the query. Finally, the Assignment Optimizer optimizes the assignment of each condition within each joining set. The rest of this section and Section 5 explain the logic of these components.

\subsection{Knowledge Administrator}

The Knowledge Administrator controls the interaction with the VO Knowledge Base, which is the component of the mediation system that maintains knowledge facts and events related to the data context of the VO. This includes intentional (schemas) and extensional information (contents) of sources. The knowledge is represented as an ontology in OWL [19] with three initial classes: VOUnit, VOResource and VODomainConcept. These classes are specialized and related to better reflect the evolution of the VO data context. VOUnit class represents the participants of the VO. An instance of VOUnit can be atomic or composite. In the latter case, it represents a temporal or a permanent group of atomic VO units working together around a specific collaboration process. VOResource class represents the physical or logical resources (like data sources) provided by VO units. Finally, VODomainConcept includes the subclasses that describe the domain of the VO. For a health VO for instance, initial classes are Patient, Medical Act, and so on.

\subsection{Benefit Estimator using Data Source Roles}

OptiSource estimates the benefit (see Definition 2) of using a source in the evaluation of a query to identify the level of relevance it will have in the query. $D S a$ is more relevant than $D S b$ if it provides more instances that match the query 
predicate, even if both of them have the same schema. In order to estimate the benefit we use the available knowledge about the extension (contents) of sources. To obtain this knowledge we work under the assumption that the organizational dimension of VOs allows to relate the role that VO units play in the VO with the contents of the sources they provide. The more complete and reliable the extensional knowledge is, the more accurate the measurement of the benefit is.

Definition 2 Data Source Benefit. Given a query $Q$ with a query predicate $P$ with conditions $\left[p_{1}, p_{2}, \ldots, p_{m}\right]$ the benefit of using a data source DSi to evaluate $Q$ is a variable that measures the degree of contribution of DSi in terms of instances that match one or more $p_{j} \in P, 1 \leqslant j \leqslant m$.

Data source roles. In a health $\mathrm{VO}$, for instance, two data sources $D S a$ and $D S b$ provide information about procedures performed on patients. Let us assume that $D S a$ is provided by a VO unit specialized on cancer whereas $D S b$ is provided by a hospital specialized on pediatric procedures. In this case, the $D S a$ may be considered specialist of patients with cancer (vo:Patient, hasDiagnosis vo:Cancer) whereas the $D S b$ is children specialist (vo:Patient, hasAge $\leq 15$ ). A source can therefore play different roles as a contributor of instances of a VDO (e.g, vo:Patient) verifying some predicate conditions (e.g, hasAge $\leq 15)$. Roles reflect the ability of sources to solve conditions. Given the analysis of the roles played by $\mathrm{VO}$ units in the business processes of the $\mathrm{VO}$, we propose the following three roles for their data sources: authority, specialist and container.

The definition of each source role is described in Definition 3,4 and 5 . In these definitions all the instances of $V D O j$ stored in data source $D S i$ are noted $\operatorname{ext}(D S i, V D O j)^{4}$. $U$ designates the data sources participating in the VO. All the instances of $V D O j$ available in the $\mathrm{VO}$ are denoted $\operatorname{ext}(U, V D O j)$. The subset of $\operatorname{ext}(D S k, V D O j)$ corresponding to the instance that verifies a condition $p$ is denoted $\operatorname{ext}(D S k, V D O j)^{p}$ and $\operatorname{card}()$ is the cardinality function.

Definition 3 Authority Role. A data source DSi plays an authority role w.r.t. a condition $p$ in a query on VDOj iff it stores all the instances of VDOj available in $U$ that match p. IsAuthority $(D S i, V D O j, p) \Longrightarrow \operatorname{ext}(U, V D O j)^{p} \subset \operatorname{ext}(D S i, V D O j)$

Definition 4 Specialist Role. A data source DSi plays a specialist role w.r.t. a condition $p$ in a query on VDOj iff most instances of VDOj stored in DSi match p. $I s S p e c i a l i s t(D S i, V D O j, p) \Longrightarrow \operatorname{card}\left(\operatorname{ext}(D S i, V D O j)^{p}\right) \geq \operatorname{card}\left(\operatorname{ext}(D S i, V D O j)^{\urcorner p}\right)$

Definition 5 Container Role. A data source DSi plays a container role w.r.t. a condition $p$ in a query on VDOj iff DSi contains at least one instance of VDOj that matches p. IsContainer $(D S i, V D O j, p) \Longrightarrow \operatorname{ext}(U, V D O j)^{p} \cap \operatorname{ext}(D S i, V D O j)^{p} \neq \varnothing$

Data source roles are registered as extensional facts in the VO knowledge base and can be acquired using three approaches: (a) Manually (e.g. expert's or DBA's definition of knowledge), (b) Interpreting the execution of processes, (c) Automatically extracting it from sources of knowledge. In [20] we have presented strategies for the last two approaches.

\footnotetext{
${ }^{4}$ This extension contains the object identifiers.
} 
Benefit model. In order to predict the benefit of a source $D S i$ w.r.t. a predicate condition $p$ the component uses Formula (1). This formula relates the role of the data source and the relative cardinality of the data source. The intention is to take into account the expected relevance of the source, given by the role factor, with the maximum number of instances that a data source may provide (in the best case). This combination of knowledge is necessary because sources that play a specialist role could be less important than sources that play container role if the number of instances the first group may provide is considerably lesser.

The RoleFactor() in Formula (2), returns a value between [0,1] indicating the most important role that a source may play in the query. ContainerFactor, SpecialistFactor and AuthorityFactor are constants reflecting the importance of the role.

$$
\begin{aligned}
& \text { Benefit }(D S i, V D O j, p)=\operatorname{RoleFactor}(D S i, V D O j, p) * \\
& \operatorname{card}(\operatorname{ext}(D S i, V D O j)) \\
& \max \{\operatorname{card}(\operatorname{ext}(D S k, V D O j)), \forall D S k \text { in } U \text { where RoleFactor }(D S k, V D O j, p)>0\}
\end{aligned}
$$

$\begin{aligned} \text { RoleFactor }(D S i, V D O j, p)=\max ( & (\text { IsContainer }(D S i, V D O j, p) * \text { ContainerFactor }), \\ & (\text { IsSpecialist }(D S i, V D O j, p) * \text { SpecialistFactor }), \\ & (\text { IsAuthority }(D S i, V D O j, p) * \text { AuthorityFactor }))\end{aligned}$

Although Formula (1) would be more accurate if the cardinality $\operatorname{card}\left(\operatorname{ext}(D S i, V D O j)^{p}\right)$ is used, the exact number of instances that satisfy a predicate is not available in VOs.

\subsection{Joining Set Manager}

To predict the set of sources that will not produce empty joins, the Joining Set Manager queries the VO knowledge base for obtaining the individuals of the composite VO units. This decision is made under the assumption that atomic $\mathrm{VO}$ units belonging to the same composite VO unit have more probability of containing the same group of instances. However, the creation of joining sets can use other type of rules to identify when two or more sources may share instances. For example, if two VO units are located in the same region, the probability that their data sources share instances may increase. Similarly, the fact that two VO units participate in the same VO process increases this probability.

Algorithm 1 uses the rule of composite units to create joining sets. The objective is to group together sources whose join will not produce an empty set and that are able to evaluate all the conditions of the query. It first [1] obtains the data sources of the composite units and creates one set for each of them. If there are redundant sets, the algorithm removes the set with fewer atomic units. Then, in [2], it validates the completeness of a set. A set is complete if the data sources it contains are able to evaluate all the conditions of the query. In [3] it extracts data sources from complete sets and [4] removes those incomplete sets whose data sources exist in the complete sets. If it is not the case, the algorithm gets the query conditions that are not already evaluated on each incomplete set 


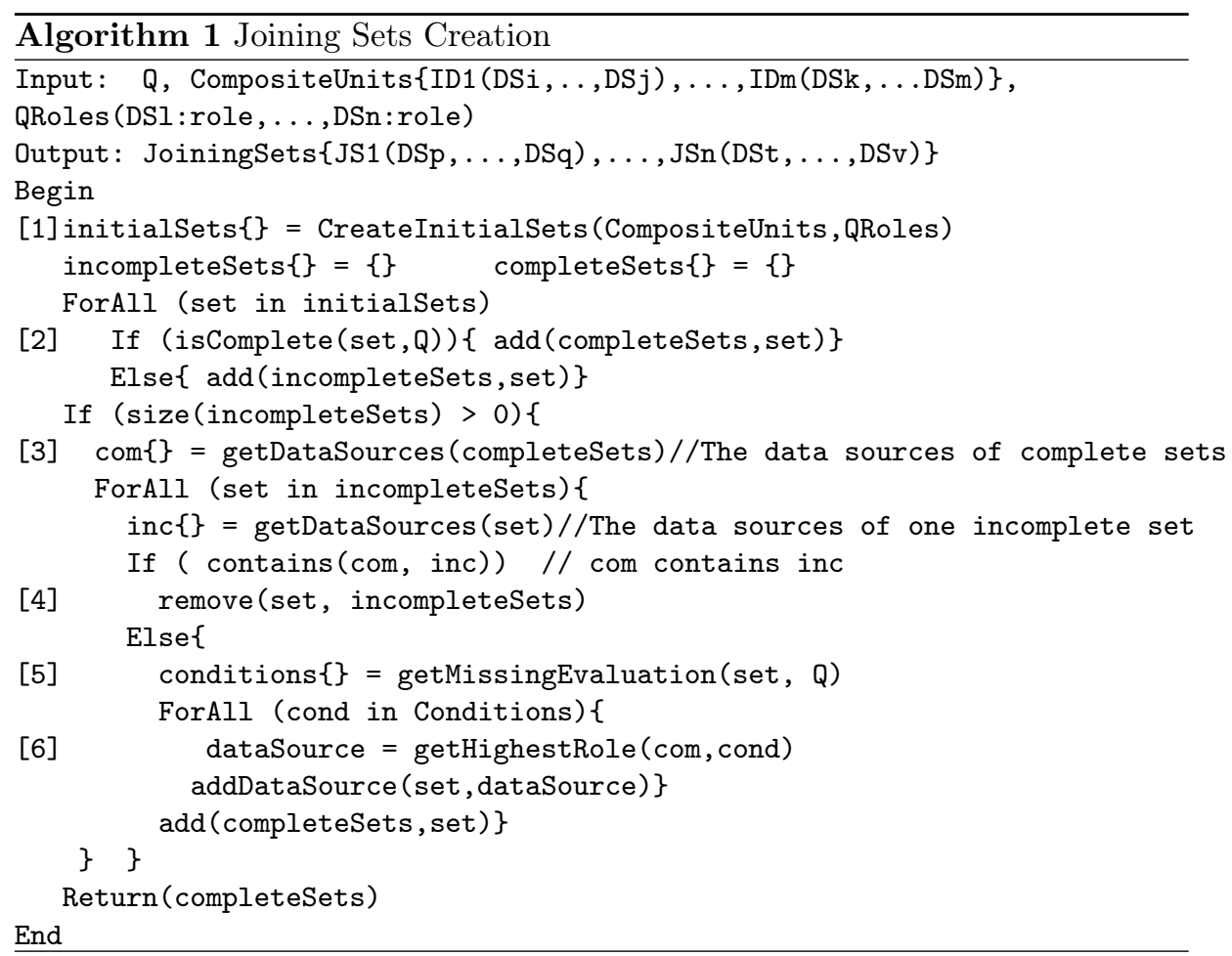

[5] and completes these sets finding [6] the data source with higher role able to evaluate the missing conditions, from the complete sets.

The output of this component is the set of joining sets. A joining set must contain at least one data source to evaluate each condition of the query predicate. However, if more than one source may evaluate the same condition, it is not straightforward to establish which one of them should be selected to evaluate the condition. It would be necessary to determine which data source assignment would improve the final answer. The Assignment Optimizer component, described in Section 5, helps to make the right decision.

\section{Optimizing Source Selection}

The proposal is to see the problem of deciding which condition predicates are evaluated by which sources as an assignment problem [21] subject to resource constraints. We propose a mathematical model that receives as input the results of the benefit predictor component and the processing capacities of sources, if they are known. Although this model was created for source selection in large scale VOs, it can be used in any distributed query processing system during the planning phase. 


\subsection{Source Selection as an Assignment Problem}

In this source selection problem, there are a number of predicate conditions to assign to a number of data sources. Each assignment brings some benefits in terms of response quality and consumes some resources of the sources. The objective is to maximize the benefits using the minimum of sources while satisfying the resource constraints. From the point of view of the predicate, one condition is assigned to one main source as main assignment, but this condition could also be evaluated as secondary assignment in parallel on other sources which have been selected by other conditions. The reason is that once a source is queried, it is better to evaluate there a maximum possible number of conditions, expecting to reduce the number of sources and the cost of transmitting instances that are not completely evaluated. Indeed, we have to deal with a bi-objective combinatorial optimization problem subject to semi-assignment and resource contraints. In practice, we choose to control the objective of minimizing the number of selected sources by converting it into a constraint. By default, the number of selected sources is limited to the number of conditions.

\subsection{Mathematical Model}

Given the set of data sources $I=\{1, \ldots, n\}$ and the set of predicate conditions $J=\{1, \ldots, m\}$, the input data are as follows :

-Ben ${ }_{i, j}$ : benefit of assigning condition $j$ to source $i, \forall i \in I, \forall j \in J$;

-MaxRes $s_{i}$ : processing resource capacity of source $i, \forall i \in I$;

-Res $s_{i, j}$ : processing resources consumed in assigning condition $j$ to source $i$, $\forall i \in I, \forall j \in J$

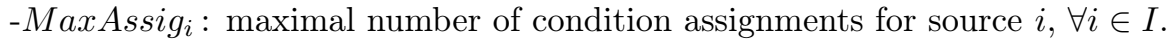

The decision variables are:

$-x_{i, j}$ are $0-1$ variables that determine whether source $i$ has $(=1)$ or not $(=0)$ been selected as a main source to evaluate the condition $j, \forall i \in I, \forall j \in J$.

$-y_{i}$ are $0-1$ variables that turn to 1 when the source $i$ is selected, $\forall i \in I$.

- assig $_{i, j}$ are 0-1 variables that determine whether a condition $j$ is assigned to source $i(=1)$ or not $(=0), \forall i \in I$. These variables represent the final assignment of conditions to sources. The $x_{i, j}$ variables indicate the main assignments while the $a_{s s i g}$ variables indicate all the main and secondary assignments.

The mathematical program of the Source Selection Optimization Problem (SSOP) can be formulated as follows :

$$
\max \sum_{j=1}^{m} \sum_{i=1}^{n} \operatorname{Ben}_{i, j} *\left(x_{i, j}+\operatorname{assig}_{i, j}\right),
$$

this objective function maximizes the benefit brought by the main and secondary source assignments of the conditions ;

subject to 


$$
\sum_{i=1}^{n} x_{i, j}=1, \forall j \in J,
$$

constraints (4) ensure that any condition $j$ is assigned to one main source $i$;

$$
\sum_{i=1}^{n} y_{i} \leq k,
$$

constraint (5) limits the total amount of queried sources to $\mathrm{k}$, we take $k=m$ by default to start the optimization process;

$$
\sum_{j=1}^{m} x_{i, j} \geq y_{i}, \forall i \in I,
$$

these constraints (6) express that if a data source $i$ is selected, there is at least one condition assigned to it, and reversely, if no condition is assigned to the data source $i$, this source is never selected;

$$
\begin{gathered}
x_{i, j} \leq \operatorname{assig}_{i, j}, \forall i \in I, \forall j \in J, \\
\text { assig }_{i, j} \leq y_{i}, \forall i \in I, \forall j \in J,
\end{gathered}
$$

these coupling constraints (7) and (8) indicate respectively that the main assignments should be in all main and secondary assignments as well, and that a source $i$ is selected when at least one condition $j$ is assigned to it;

$$
\begin{gathered}
\sum_{j=1}^{m} \operatorname{Res}_{i, j} * \operatorname{assig}_{i, j} \leq \operatorname{MaxRes}_{i}, \forall i \in I, \\
\sum_{j=1}^{m} \operatorname{assig}_{i, j} \leq \operatorname{MaxAssig},, \forall i \in I,
\end{gathered}
$$

these resource constraints (9) and (10) ensure that all the main and secondary assignments of conditions do not exceed neither the processing resource capacities nor the maximum number of possible assignments per source.

Notice that constraint (5) is somehow redundant with constraint (6) which prevents to select a source if no condition is assigned to it, i.e. the number of selected data sources is always less than or equal to the number of conditions $m$. But in practice, one could reduce $k$ in constraint (5) to control the minimization of the number of selected sources.

The resolution of the model provides the selected sources in $y_{i}$ variables and all the main and secondary assignments in $a_{s s i g} i_{i, j}$ variables. The joining of the results provided by each condition are the instances required by the user. If the number of instances obtained from the joining sets are not enough to satisfy user requirements, the query planner will use the model to generate a new assignment with the remaining sources (i.e. those that were not in $y_{i}$ ). 


\subsection{Theoretical Complexity and Solving Methods}

The Source Selection Optimization Problem (SSOP) is a knapsack-like problem. If we consider only the $a_{s s i g}, j$ variables and their related constraints, the problem is similar to a two-dimensional multiple knapsack problem which is NP-hard since it is a generalization of the knapsack problem. We have two types of resource constraints (9) and (10) for each data source (two dimensions for each knapsack) and multiple data sources (multiple knapsacks).

However, there are two differences. The first one is that one condition (item) is assigned to (i.e. evaluated by) one main data source (constraints (4)) and can be assigned also to several secondary data sources. The semi-assignment constraints (4) are only on the main assignment variables $x_{i, j}$. The second one is that we have to control the number $k$ of the data sources selected via the variables $y_{i}$ and constraint (5). This is similar to contraints of the uncapacitated facility location problem.

Indeed, if we consider the version of the SSOP in which we look only for the main assignements (more restricted than SSOP), denoted MSSOP, the MSSOP can be easily transformed into a multi-dimensional multiple knapsack problem which is NP-hard. Since the resolution of the MSSOP provides a feasible solution to the SSOP, the SSOP is also NP-hard.

To solve the SSOP, we have chosen to use in this study two well-known MIP solvers: CPLEX and GLPK, because the computational times obtained on the test data instances are acceptable. Naturally, heuristics and metaheuristics can be used to speedup the optimization if computational time constraint is tight and exact solutions are not required. In addition, heuristics and metaheuristics are also more suitable if multi-objective optimization is considered in this problem, i.e. altogether maximizing the benefit of the condition assignments to the data sources and minimizing the number of data sources selected.

\section{Implementation and Validation}

In order to evaluate the behavior of OptiSource and validate its improvement on the selection of the most relevant data sources, a prototype of OptiSource has been constructed and used to evaluate its precision and recall. This section presents the main results obtained during this evaluation. Section 6.1 presents the characteristics of the prototype. Section 6.2 details the experimental context. Section 6.3 gives the evaluation using different data contexts and levels of knowledge about sources. Finally, Section 6.4 compares our strategy to related works.

\subsection{Prototype}

OptiSource is part of a mediation system created for VOs. For evaluating the behavior of OptiSource we developed the components of OptiSource presented in Figure 7 as well as other components required for the query planning. The complete set of components of this prototype are presented in Figure 8. OptiSource is the SourceSelector component. 


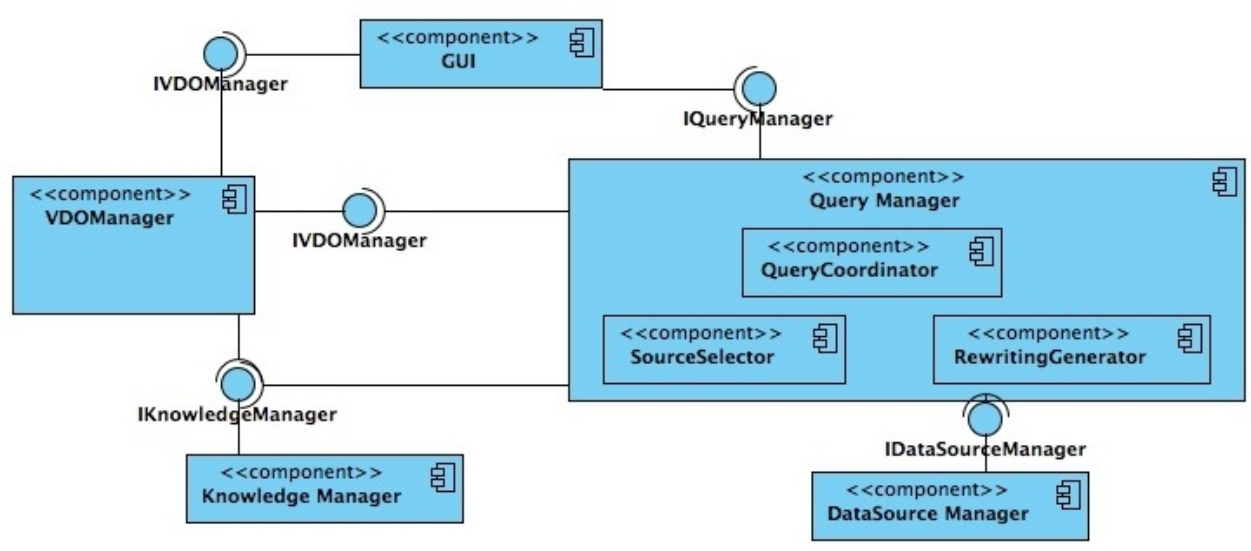

Fig. 8. Prototype Components.

Components are written in Java. The knowledge base is implemented in OWL [19]. The optimization model is written in GNU MathProg modeling language and is processed by the GNU Linear Programming Kit (GLPK) [22]. We also validate our optimization model performance in CPLEX 10.2. For the implementation we use the Java Binding for GLPK called GLPK-Java [23]. Queries of VDOs are accepted in SPARQL [24] and are processed using Jena API and Pellet as the inference engine.

\subsection{Experimental Context}

To measure the extent to which the selection made by OptiSource is successful, it has been assessed whether OptiSource choose the data sources in order of relevance to the query. The evaluation has been conducted using evaluation measures that are typically applied in information retrieval systems, applying certain adjustments to the context. Figure 9 presents the sets of data sources that have been controlled during the experiments. The Extensional Relevant Data Sources are the sources that have at least one instance that matches the query predicate. The Relevant $k$ Data Sources are the $k$ data sources that have the higher contribution in terms of number of instances that match the query predicate, where $k$ varies in each experiment.

The experiments are focused on measuring three metrics: Extensional Precision, that measures the extensional relevant data sources selected from the total set of data sources selected; $k$ Precision, that measures the $k$ relevant data sources selected from the total set of data sources selected; and the $k$ Recall, that measures the first $k$ data sources selected from the total set of $k$ relevant data sources. 


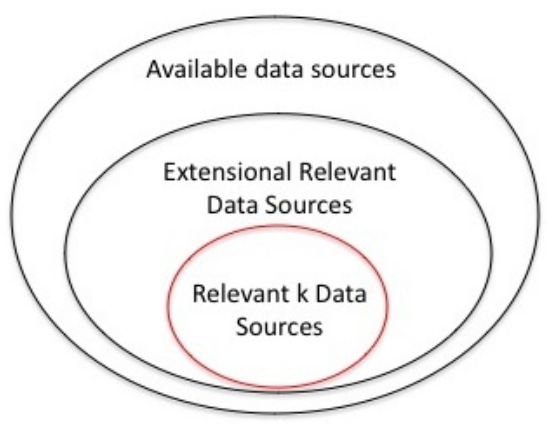

Fig. 9. Experimental Sets.

To obtain these metrics, we measure the following variables:

Rext: Data sources relevant extensionally for the query Q.

$\boldsymbol{R} \boldsymbol{k}$ : First k data sources relevant extensionally for the query $\mathrm{Q}$.

$\boldsymbol{A}$ : Data sources selected by OptiSource.

$\boldsymbol{A} \boldsymbol{k}$ : First k data sources selected by OptiSource.

The formulas used to calculate each metric are the following:

Extensional Precision: $|A \cap \operatorname{Rext}| /|A|$.

k Precision: $|A k \cap R k| /|A k|$.

k Recall: $|A k \cap R k| /|R k|$.

Experimental scenarios vary in three dimensions: the number of sources, the level of knowledge of sources and the relationship between the query and the data context. The number of sources can vary between 30 to 1000 data sources. The level of knowledge represents the precision and completeness of the knowledge facts included in the knowledge base w.r.t. the query. Changes on the relationship between the query and the data context cover the worst case of distribution where the query answer is distributed over more than $80 \%$ of data sources, and the best case where the answer is concentrated in less than $20 \%$ of data sources.

We define the knowledge base of a VO in the health domain that shares information about patients. Metadata are generated at different levels of the knowledge base using a developed generator of metadata in XML. This generator describes the intentional knowledge of data sources, the known roles of each data source and possible replication of instances with other data sources. For instance, a data source may declare: < class name ="Glaucoma" role="Container" $>$, and another one can declare $<$ class name $=$ "OperationontheEye" role="Container" $>$.

\subsection{Experimental Results}

Due to space limitations we present only the most significant portion of the experiments executed. Figures 10 and 11 illustrate the impact of level of knowledge changes on the precision and recall of OptiSource. Low level means the knowledge base only contains the intentional knowledge of sources (e.g. can evaluate 


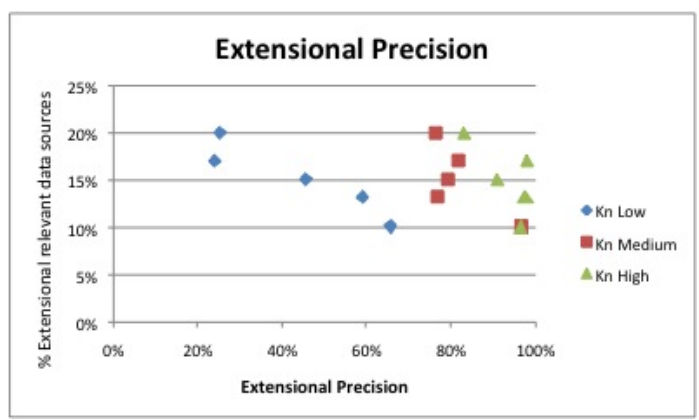

Fig. 10. Extensional precision evaluation changing the level of knowledge.

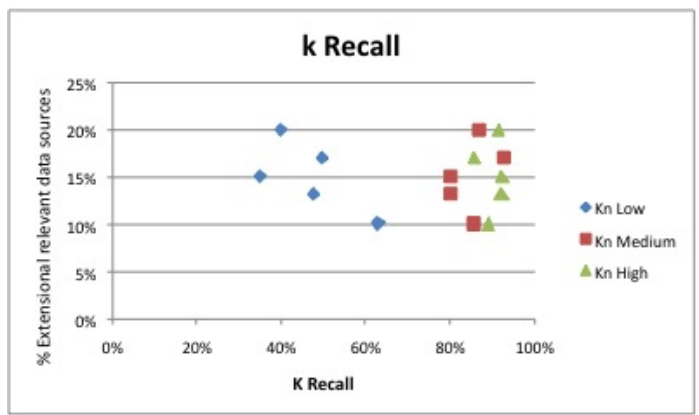

Fig. 11. k Recall evaluation changing the level of knowledge.

the diagnosis). The medium level means it also knows a group of roles of sources related to classes of the domain concept, but they are related to higher classes of the knowledge base. For instance, sources have related roles to cancer and queries ask for kidney cancer. High level indicates that roles are related to more specific classes (e.g. roles related to kidney cancer).

The experiments show that even if a high level of knowledge of the context is desired, OptiSource precision and recall have a good behavior with a medium level. This is due to the fact that a medium level of knowledge is enough to discard a large group of sources and to direct the queries to sources with higher probabilities of having matching instances. Additionally, and because of the use of an ontology to represent the knowledge base, even if the knowledge facts are not expressed at the same level of the query conditions, the inference engine infers knowledge facts that enhance the estimation of the benefit.

Figure 12 presents the impact of the relationship between the query and the data context on the extensional precision and the $\mathrm{k}$ precision. It also compares the results with a strategy oriented to capabilities. Figure 12 illustrates the behavior of OptiSource when the instances are concentrated in less than $20 \%$ of the available data sources. In such cases OptiSource exceeds by more than $50 \%$ the extensional precision and the k precision of the capability oriented strategy. 

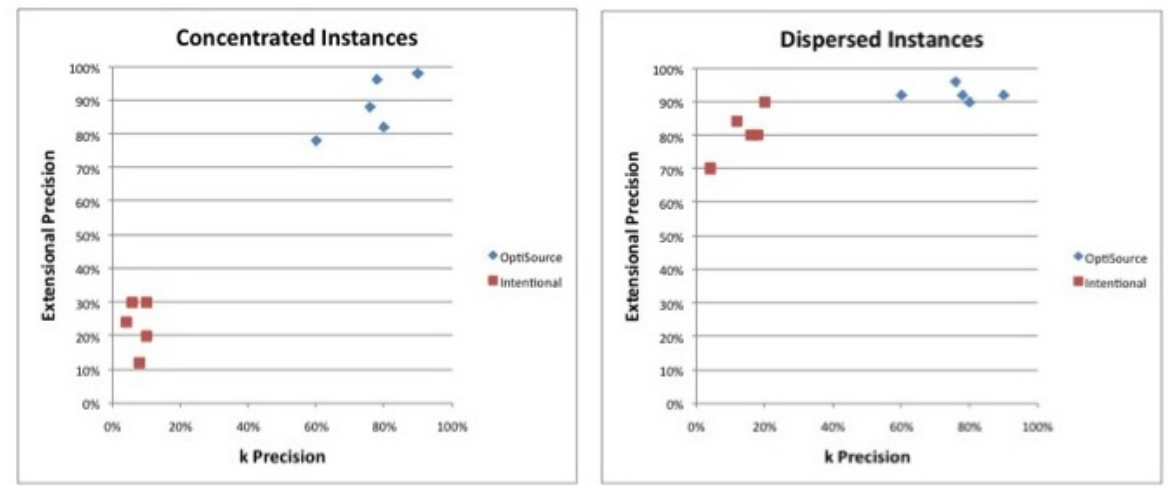

Fig. 12. Extensional precision and $\mathrm{k}$ precision evaluation changing the relationship between the query and the data context.

This is the result of source reduction performed by OptiSource using the roles and the cardinality of data sources. OptiSource precision is stable and always higher than the strategies that only use intentional knowledge and capabilities.

Tests also show that for data contexts with fewer data sources, the improvement in precision according to the level of knowledge is not significant. This validates our assumption that in small data contexts great efforts in query planning for reducing queried sources are not necessary. Still, the improvement observed when there is a large number of sources led us to conclude that OptiSource is especially useful under these circumstances.

Finally, the experiments show that in the worst case (low level of knowledge) OptiSource selects the same set of sources that traditional strategies of query rewriting select because it only considers the intentional views of sources. Other experimental results related to the precision and recall of OptiSource are presented in [9].

\subsection{Comparison with Related Works}

Although OptiSource cannot be directly compared with any of the proposals in the literature, we evaluated the implications of using two available strategies (QPIAD [6] and Navigational Paths [14]) in VO contexts. We also adapted the proposal iDrips [5] since it is the closest to OptiSource in its proposed intention.

The strategy of rewriting of QPIAD [6] allows the query processor to return certain and "possible" certain answers from databases. The first type are answers that match all the query predicate; the second type are the answers that probably match the query, but have one condition that cannot be evaluated because the required value is missing ( $n u l l$ ). QPIAD generates and uses detailed statistics on source's quality to reformulate a query according to the characteristics of each source that is able to evaluate the predicate. Even though the principle of QPIAD will be very attractive for use in VOs whose sources have 
quality problems, the strategy could not scale up when the number of sources is large. To prove this we will consider a VO with 20 sources, which are able to evaluate a query $\mathrm{Q}$ in terms of the schema. We supposed that in average each source provides 500 certain answers. For each source 50 query reformulations are required to obtain the possible answers. This means that $1020\left(50^{*} 20+20\right)$ queries are executed including the original $\mathrm{Q}$ to obtain certain answers from each source.

Another strategy connected to VO contexts is the proposal to improve the selection of navigational paths between biological sources [14]. It assumes the availability of a graph describing the objects contained in the sources and their relationships. The objective is to find the path with best benefit and lower cost given an initial and a final object class. Applying this proposal to our problem we found that it is possible to organize sources in a graph. Each node could contain sources with equivalent intentional fragments of VDO, and the relationships between sources that contain the missing fragments of VDO could be the links between different nodes. For instance, if sources 1 and 2 know the Demographic Data and Medical Act classes of Patient they will be in the same node, and will be related to nodes that contain sources that know the Affiliation class. Using this graph it will be possible to select the most "profitable" sources for a query; however, it is not clear how the benefit of each path is obtained and how the overlapping between each path can be calculated. This lack could be combined with our strategy using the roles of data sources in each path to compute its potential benefit. Thus, the prediction model of OptiSource can be used to compute the ratios between paths used in this proposal.

Finally, we compared OptiSource with the iDrips algorithm. This comparison is focused on measuring the recall in terms of relevant instances obtained vs. the number of sources queried. We did not measure precision because iDrips and OptiSource always return correct instances. In order to make comparable the measurements of recall in OptiSource and iDrips, we evaluate in each iteration of iDrips the number of relevant instances until it finds the complete set of relevant instances. We also measure the number of queried sources in each iteration. In the case of OptiSource, we measure the relevant instances obtained after the execution of subqueries of the joining sets.

We used the plan coverage as the utility measure for iDrips and three formulas of utility that led us to analyze the different levels of knowledge of the VO context. Given a plan $P=\{D S 1, \ldots, D S n\}$ where $D S i$ are the sources that will be queried on the plan and a query $Q=\left\{p\left(p_{1}, \ldots, p_{m}\right)\right\}$ with m predicate conditions, the three formulas to compute plan coverage are as follows:

$$
\operatorname{Cov}(P)_{T 1}=\min (\operatorname{ext}(D S i, V D O j)) \text { where } D S i \in P \text {, }
$$

this formula assumes that the number of instances of the VDO sources contain is known;

$$
\operatorname{Cov}(P)_{T 2}=\min (\operatorname{overlap}(D S i, D S k)) \text { where DSi andDSk } \in P,
$$

this formula assumes the same knowledge of (11) and the level of overlapping between data sources; 


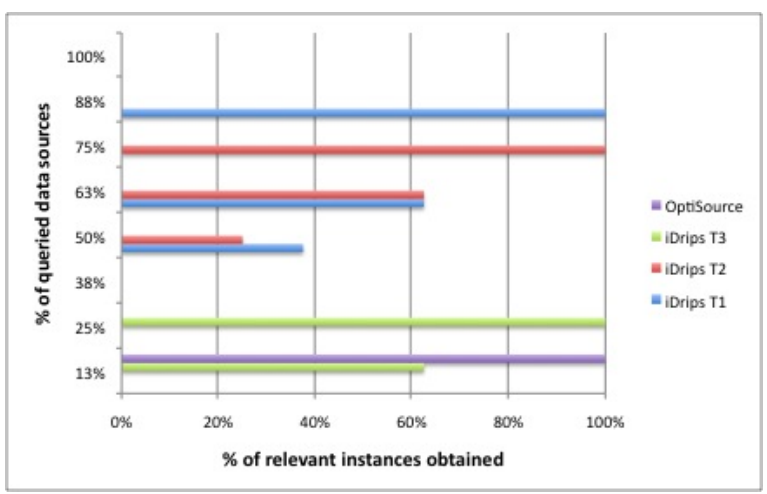

Fig. 13. OptiSource vs. iDrips.

$$
\operatorname{Cov}(P)_{T 3}=\frac{\operatorname{ext}(D S i, V D O j)^{p}-\left(\operatorname{ext}(D S k, V D O j)^{p} \ldots \operatorname{ext}(D S l, V D O j)^{p}\right)}{\operatorname{ext}(U, V D O j)^{p}}
$$

where $D S i, D S k$ and $D S l \in P$ and $D S k \ldots D S l$ have been already queried in a previous plan. Formula (13) assumes the knowledge of (11) and (12). It also requires knowing the number of instances that satisfy the query predicate in each source (or in each plan) and from these instances which ones can also be provided by another plan. Even though this information will be difficult and expensive to obtain in a VO context, we used it because the creators of iDrips assumed having it when they designed the algorithm.

The first group of experiments are run using a set of synthetic sources that are extensionally and intentionally overlapped, and where a few number of sources contain a large number of relevant instances; even though instances are distributed largely along the VO sources. In other words, in these contexts there are sources specialized in one or more of the query conditions. Consequently, querying them is enough to obtain almost all the relevant instances. iDrips is evaluated using the aforementioned utility measures. Figure 13 illustrates the differences on recall between iDrips and OptiSource. The y axis shows the percentage of queried sources from the total number of relevant data sources. On the other hand, the $\mathrm{x}$ axis illustrates the percentage of instances obtained.

The results demonstrate the effectiveness of OptiSource in this type of contexts where it only queried $13 \%$ of relevant sources to obtain $100 \%$ of relevant instances. On the contrary, iDrips required to query $25 \%$ of relevant sources to obtain the $100 \%$ of relevant instances in the best case (using Formula (13)). Figure 13 shows that the number of sources queried by OptiSource is considerably lower than those queried using (11) and (12) of the plan coverage. Although this could be inferred considering the difference on the level of knowledge, it allows us to conclude that solutions for ranking queries cannot be applied to VOs without using a powerful utility function able to represent source relationships and source probability of having query relevant instances. Nonetheless, even with strong utility functions, the absence of replication knowledge prevents 
discarding sources that do not contribute with new relevant instances. This lack affects iDrips when we use Formula (13).

We have also run experiments in a context where synthetic data sources are overlapped intentionally but not extensionally, and a small percentage of sources are specialized in one or more of the conditions of the predicate. As expected, the behavior of both strategies are similar because it is necessary to contact almost all the sources related intentionally to the query.

\section{Analyzing the Impact of Prediction Accuracy}

The most important input of the optimization model is the benefit of using a data source to evaluate a query condition. As it has been illustrated, the value of the benefit is predicted taking into account the role of the data source and its relative size. If the prediction is well done the optimization will be very accurate. However, if there is not certainty about the validity of the prediction, the model will probably produce inaccurate results. Thus, this section presents first the methodology used to evaluate the impact of the benefit value in the final assignment, then it presents the obtained results and an analysis over them.

\subsection{Analysis Methodology}

Sensitivity analysis of optimal solutions is a well-known task that allows to evaluate the impact of changes in input data parameters of linear programming models. Although there are well-known methods to evaluate the sensitivity of the optimization model results, due to the high degeneracy of the assignment problem, these traditional methods are impractical in our case [25]. Additionally, even though there are some proposals to determine the sensitivity range of parameters in the assignment problem [25], they could not be applied to our model because they assumed the use of the classical assignment problem, and although our model is close to the assignment problem, it differs from the classical one by its resource constraints. Besides, they assumed the unimodular property of constraint matrix, which is not checked by the matrix of our model. Consequently, in order to evaluate the impact of the model, we set up an experimental study framework, which allows us to measure the impact of the benefit values. The idea behind is to measure changes on the assignment when the value of the benefit values are known under uncertainty.

To ensure that the experiments are significant, the model is applied to different sets of data that change the number of data sources $(25,50,100,200,400,800)$ and the number of conditions $(5,10,20)$; at the end 18 sets are produced. For each one of the sets, a benefit matrix is created $\left(B e n_{i, j}\right.$ in Section 5$)$ with real values: this matrix is called the initial matrix. Using this initial matrix the proposed model is executed using GLPK. The assignment obtained from the execution of the model for each set is considered as the base result for each set.

The initial matrix is then modified in order to simulate the uncertainty of the value of each benefit. The precision of the value is reduced randomly, i.e. the new values of $B e n_{i, j}$ can vary from $0.33 \times B e n_{i, j}$ to $0.66 \times B e n_{i, j}$. The 


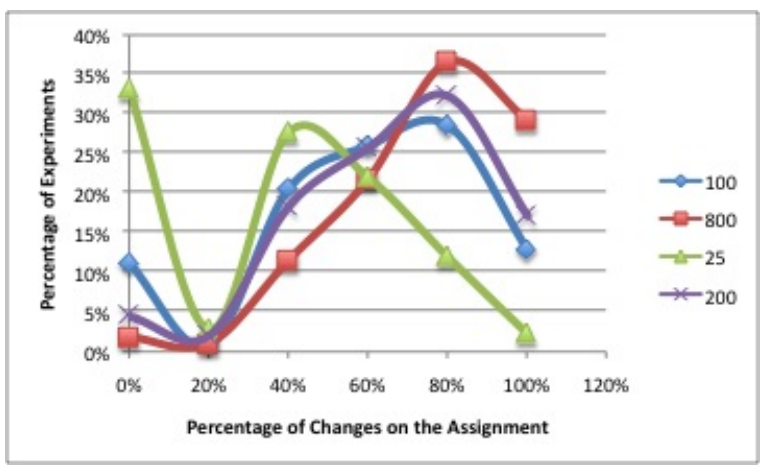

Fig. 14. General Impact of the Benefit.

intention of these reductions is to change the value of the benefit in a similar way as it is reduced when the role of a data source or the size of a data source is not known precisely. These new matrices produce secondary results that are compared with the base results. For each initial matrix, 50 new matrices are generated for each set.

\subsection{Experimental Results}

Figure 14 illustrates a summary of the experiment results. The $\mathrm{x}$ axis represents the percentage of assignments that change in an experiment. The y axis represents the percentage of experiments for which it has been obtained a change in the assignment. Each line represents the number of data sources in the experiment. For example, with 100 data sources, $11 \%$ of experiments did not have any change on the assignment when the benefits are changed. Similarly, with 800 data sources $22 \%$ of experiments had $60 \%$ of changes on the assignment when the benefit changes. As it can be noticed, changes variability is directly related to the number of data sources. This result has been expected because the higher the number of data sources is, the greater the probability of finding a data source with a better profit value that forces to change the assignment is. The study also demonstrated that most part of the experiments had changes in the assignment between $40 \%$ and $70 \%$. Besides, the tests show that $25 \%$ of the assignments had changes of $60 \%$ independently on the number of data sources. Figure 15 shows the average of experiments for each set of changes. For example, $27 \%$ of experiments had changes near $60 \%$, and $18 \%$ of experiments had changes near $40 \%$.

Figure 16 and Figure 17 present the percentage of changes when the benefit was changed a maximum of $15 \%$ or a maximum of $50 \%$, respectively. As in the general case, most part of experiments had assignment changes between $40 \%$ to $70 \%$, independently on the percentage of changes on the benefit value.

Results on sensibility show that changes on the values of the benefit, as expected, affects the final response. However, a percentage from $20 \%$ to $60 \%$ 


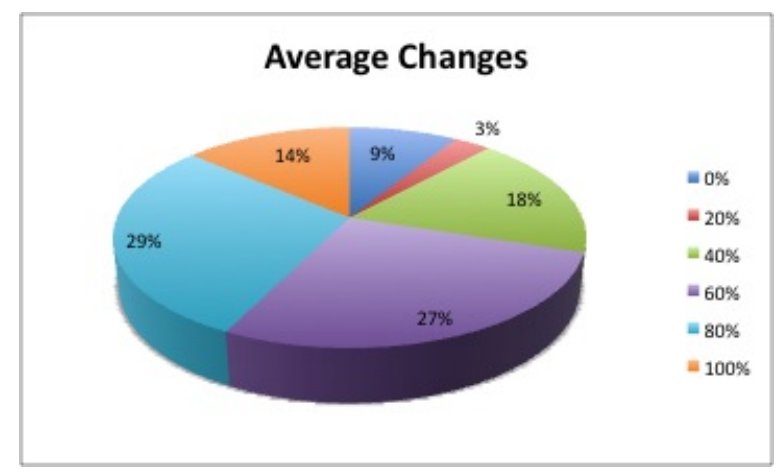

Fig. 15. Average of changes in all the experiment sets.

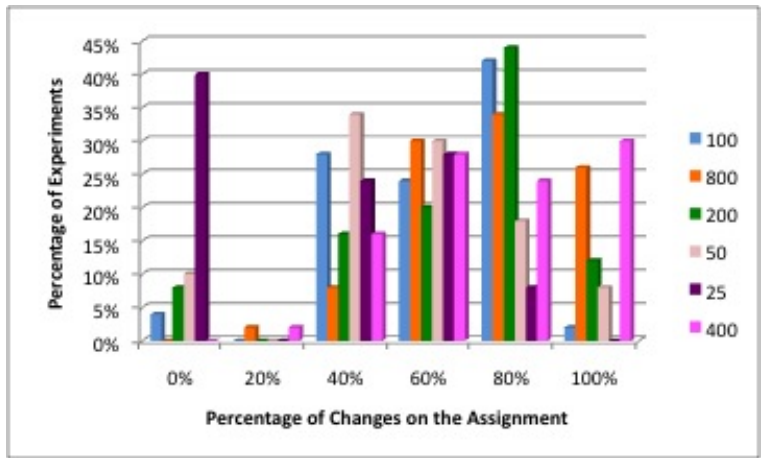

Fig. 16. Impact of the Benefit in the Assignment - $15 \%$.

of the assignments remain the same, even if the values of the benefit changes $50 \%$ from the base value. This fact, demonstrates a good level of robustness of our optimization model, which does not require a precise prediction in order to make an assignment near to the optimal.

\section{Conclusions and Future Work}

Virtual Organizations including a large number of autonomous participants that collaborate to share knowledge are very common in web contexts. The number and autonomy of the participants prevent a global understanding of the data characteristics and their relationships. This leads to complex data contexts where query processing is expensive. One of the main factors for that is the difficulty to effectively select the sources to access for a query evaluation. As current strategies do not focus on contexts with numerous heterogenous overlapping sources their use in large scale VOs will lead to the generation of redundant queries to unnecessary sources. 


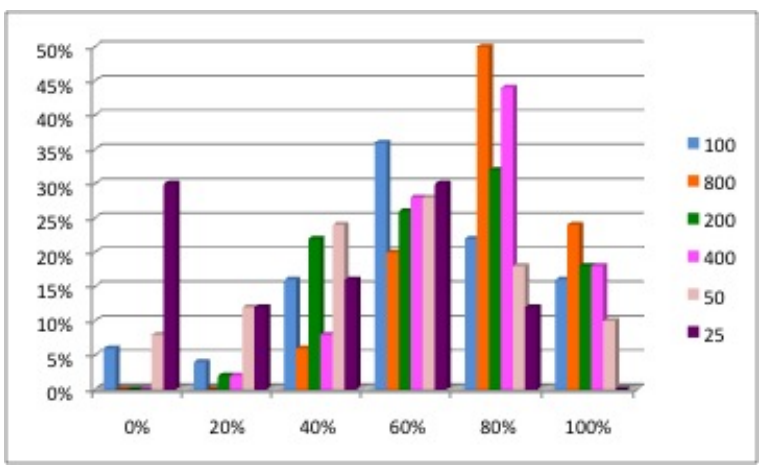

Fig. 17. Impact of the benefit in the assignment - 50\%.

In order to tackle this difficulty, we have modeled the source selection problem as a complex decision problem using combinatorial optimization techniques. This leaded us to combine several original contributions including knowledge representation and a mathematical formulation of the source selection problem.

This paper presented the main choices and OptiSource, the proposed source selection strategy. It is based on the prediction of the benefit of using a source during query evaluation according to its individual and group relevance. The combinatorial optimization model is used to select the most relevant sources to be used to evaluate each query condition. The main choices and contributions are the following:

- The context is represented with a generic ontology that can be specialized according to the problem domain. This ontology facilitates the description of a VO. We proposed a general representation including VOUnits, VOResources, VODomainConcepts and their relationships. This enhances the knowledge of the mediator and facilitates its evolution if necessary.

- We proposed the use of roles for defining relationships between sources, concepts and queries. Roles represent a hierarchical relationship between sources and concepts that enable the source prioritization during the source selection phase. The roles presented in this paper are inspired by a health domain. Nevertheless, our proposal is generic and the roles can be customized according to the application domain. Roles contribute to the estimation of the individual contribution of a source to evaluate a query predicate.

- OptiSource adopts a combinatorial optimization model that establishes which sources evaluate each condition of the query. This model uses the knowledge about data sources and their relationships. The discovery process of such knowledge is based on Virtual Organization description (such as composite VO Units), their business processes, geographical location of participants among others. Additional relationships between participants can 
be expressed by experts or deduced from historical query process.

- A prototype of OptiSource has been developed in Java and used GLPKJava. The knowledge base is implemented in OWL. The optimization model is written in GNU MathProg modeling language and is processed with the GNU Linear Programming Kit (GLPK). CPLEX 10.2 has also been used to validate the combinatorial optimization model. Queries of VDOs are accepted in SPARQL and are evaluated using Jena API and Pellet as the inference engine.

- Numerous experiments have been conducted, in particular to evaluate the precision and the robustness of our proposal. Experiments have demonstrated that OptiSource is effective to reduce the contact of unnecessary sources when data contexts involve extensional and intentional overlapping. They also showed that OptiSource is an evolving system whose precision grows according to the degree of knowledge of sources, but does not require a high level of knowledge to have good levels of precision and recall. Sensibility analysis of OptiSource w.r.t. the benefit estimation demonstrates a good level of robustness of the optimization model, which does not require a precise prediction in order to make an assignment near to the optimal.

This work opens important research issues. Among them, the extension of the work of extracting and using Virtual Organization knowledge to improve data management. The use of data mining techniques on logs of business processes and query executions is promising. The combination of such knowledge with statistics should also be further developed. Future research also involves the use of our proposal in other contexts leading probably to other source relationships and data source roles.

To finish, probably the most important research perspective we consider, is the further use of combinatorial optimization techniques for the optimization of large scale information systems, such as multi-objective optimization and metaheuristic solving methods to enable more complex modeling of the source selection optimization problem. Our experience is very positive and we intend to pursuit this approach.

\section{References}

1. Foster, I., Kesselman, C., Tuecke, S.: The anatomy of the grid: Enabling scalable virtual organizations. International Journal of High Performance Computing Applications 15 (2001) 200-222

2. NEESGrid: Nees consortium, http://neesgrid.ncsa.uiuc.edu/ (2008)

3. BIRN: Bioinformatics research network, http://www.loni.ucla.edu/birn/ (2008)

4. Quiané-Ruiz, J.A., Lamarre, P., Valduriez, P.: Sqlb: A query allocation framework for autonomous consumers and providers. In: VLDB. (2007) 974-985

5. Doan, A., Halevy, A.Y.: Efficiently ordering query plans for data integration. In: ICDE '02, Washington, DC, USA, IEEE Computer Society (2002) 393 
6. Wolf, G., Khatri, H., Chokshi, B., Fan, J., Chen, Y., Kambhampati, S.: Query processing over incomplete autonomous databases. In: VLDB. (2007) 651-662

7. Huebsch, R., Hellerstein, J.M., Lanham, N., Loo, B.T., Shenker, S., Stoica, I.: Querying the internet with pier. In: VLDB. (2003) 321-332

8. Pottinger, R., Halevy, A.Y.: Minicon: A scalable algorithm for answering queries using views. VLDB Journal. 10 (2001) 182-198

9. Pomares, A., Roncancio, C., Cung, V.D., Abásolo, J., Villamil, M.D.P.: Source selection in large scale data contexts: An optimization approach. In: DEXA. (2010) 46-61

10. Levy, A.Y., Rajaraman, A., Ordille, J.J.: Querying heterogeneous information sources using source descriptions. In: VLDB. (1996) 251-262

11. Garcia-Molina, H., Papakonstantinou, Y., Quass, D., Rajaraman, A., Sagiv, Y., Ullman, J.D., Vassalos, V., Widom, J.: The tsimmis approach to mediation: Data models and languages. Journal of Intelligent Information Systems 8 (1997) 117-132

12. Tomasic, A., Raschid, L., Valduriez, P.: Scaling access to heterogeneous data sources with DISCO. Knowledge and Data Engineering 10 (1998) 808-823

13. Yerneni, R.: Mediated Query Processing Over Autonomous Data Sources. PhD thesis, Stanford University, Stanford, CA (2001)

14. Bleiholder, J., Khuller, S., Naumann, F., Raschid, L., Wu, Y.: Query planning in the presence of overlapping sources. In: EDBT. (2006) 811-828

15. Naumann, F., Freytag, J.C., Leser, U.: Completeness of integrated information sources. Information Systems Journal, Special issue: Data quality in cooperative information systems 29 (2004) 583-615

16. Tatarinov, I., Ives, Z., Madhavan, J., Halevy, A., Suciu, D., Dalvi, N., Dong, X.L., Kadiyska, Y., Miklau, G., Mork, P.: The piazza peer data management project. SIGMOD Rec. 32 (2003) 47-52

17. Nejdl, W., Wolf, B., Qu, C., Decker, S., Sintek, M., Naeve, A., Nilsson, M., Palmér, M., Risch, T.: Edutella: a p2p networking infrastructure based on rdf. In: WWW '02, New York, NY, USA, ACM (2002) 604-615

18. Adjiman, P., Goasdoué, F., Rousset, M.C.: Somerdfs in the semantic web. Journal on data semantics 8 (2007) 158-181

19. Horrocks, I.: Owl: A description logic based ontology language. In: Principles and Practice of Constraint Programming. (2005) 5-8

20. Pomares, A., Roncancio, C., Abasolo, J., del Pilar Villamil, M.: Knowledge based query processing. In: ICEIS. Volume 24 of Lecture Notes in Business Information Processing., Springer (2009) 208-219

21. Hillier, F.S., Lieberman, G.J.: Introduction to Operations Research. 8th edn. McGraw-Hill (2005)

22. Makhorin, A.: Gnu project, gnu linear programming kit. http://www.gnu.org/software/glpk/ (2009)

23. Makhorin, A.: Gnu project, glpk for java. http://glpk-java.sourceforge.net/ (2009)

24. Eric Prud, A.S.: Sparql query language for rdf, http://www.w3.org/tr/rdf-sparqlquery/ (2007)

25. Lin, C.J., Wen, U.P.: Sensitivity analysis of the optimal assignment. European Journal of Operational Research 149 (2003) 35-46 